\title{
Testing the broadband network : a quickly shifting demand for test equipment solutions
}

\author{
Ing. L. Vandevenne \\ Project Manager
}

Siemens Atea, Atealaan 34, B-2200 Herentals

e-mail :p82453@vnet.atea.be

Ir. B. De Sutter

Broadband, Networks and Protocols

Siemens Atea, Atealaan 34, B-2200 Herentals

e-mail : p82581@vnet.atea.be

Ing. E. Goossens

Verification Testing and Validation

Siemens Atea, Atealaan 34, B-2200 Herentals

e-mail:p82835@vnet.atea.be

\begin{abstract}
In the area of test needs there is a quickly shifting demand for test-equipment. This is initiated, not only by the new transmission techniques (such as ATM), but also by network sizes. Nowadays national specific variants loose interest, and cross-border standardizations result in more transparant and cost-effective telecommunication networks.

This paper describes the research results of a project of Siemens Atea in the area of test needs. It has mainly two segments : the first describes the test world where signaling protocols are involved, and its segmentation. The second part describes a model of a solution. It contains also some of the software design techniques used for designing a prototype.
\end{abstract}

\section{Keywords}

testing, ATM, broadband, B-ISDN, telecommunication, protocols, interfaces 


\section{INTRODUCTION}

The booming demand for communication bandwidth has created the need for broadband networks. The established base of narrowband technology is unable to cope with the exponentially increasing data throughput, which is to be delivered by networks intended for multimedia applications, requiring high- capacity video-, audio- and data-streams.

Within this rapidly evolving domain of broadband networking, the phrase "Asynchronous Transfer Mode" has truly become a cliché. The entire communications industry seems to be aware of the major advantages offered by the ATM technology. The main benefits can basically be subdivided into 3 categories:

- ATM can support a large variety of integrated applications requiring large and flexible bandwidths.

- ATM is scaleable and can be placed on virtually any type of physical carrier, ranging from low-end $2 \mathrm{Mbit} / \mathrm{s}$ copper links (E1/DS1) up to multi-Gbit/s optical fiber.

- ATM offers highly efficient and easily manageable networking capabilities.

However, these advantages are haunted by the inevitable ghost of the "testing netherworld". Clearly, testing and troubleshooting this kind of high performance ATM-based networks is not a task to be taken lightly. Human abilities to control and observe the immense amount of exchanged data involved are totally insufficient.

Therefore, the purpose here is not to elaborate on the "why" and "how" of broadband network design and evolution, but to present a point of view on how these networks can be tested without having to deal with a mental test hangover.

\section{DEFINING THE KEY TEST ELEMENTS}

Power and flexibility are not the only players in the game to ensure that the ATM-based network technology will be successful. In order to check and guarantee their stability and reliability in network environments, ATM systems have to be subjected to extensive testing at all levels, starting from the design and implementation phases up to daily under-operation checking and trouble-shooting.

ATM is destined at making its appearance in network solutions of varying scale, from local corporate utilization up to pan-European or even global interconnected high-performance networks. This implies automatically a multi-vendor, multi-operator environment. As a result, ATM network testing activities have to take these abilities into account.

Globally summarizing the common factors within any test process, results in following elements:

- A testable entity.

- A selection of properties to be tested.

- suitable test tools 
The scope and purpose of the test processes can differ significantly. What we want to do here, is to define the above listed key elements for ATM network-related testing.

\subsection{A testable entity}

Depending on the desired scope of testing, this can either be:

- a single ATM access or network node (= switch or concentrator)

- multiple, inter-operable ATM network nodes (= the network itself)

The tests carried out on the single ATM switch are essential for the manufacturer or vendor involved. It allows them to verify their system during the design and implementation phase, in an environment where failures or bugs cannot influence a critical operational network. The network operator can then decide, afterwards, to perform a series of acceptance tests on this single switch, also without endangering the already established network.

Once a stable system is integrated into the ATM network, additional day-to-day operational tests remain necessary for maintenance or troubleshooting purposes. These tests can only be carried out within the (potentially global) operational network, thereby possibly triggering the need for efficient remote testing.

\subsection{A selection of properties to be tested}

The core of each test process consists of the system or network properties that need to be tested. The earlier mentioned power and flexibility, which ATM technology brings to broadband switching systems and networks, certainly do not reduce the number of test paths and interworking functionalities to be covered.

The most important properties that have to be subjected to testing in ATM networks are the following:

\section{Signaling protocols:}

Certainly the primary aim in any switched ATM network test. The presence of signaling is an absolute prerequisite in any network. Many protocols can, conform to the OSI concept of layers, ride on the ATM protocol. Although bodies like ITU and ATM Forum define standards, such as the ITU-Q.2931 in case of UNI-signaling, the appearance of variants cannot be avoided. These variants bring incompatibility problems along, which are impossible to trace without decent testing and monitoring.

\section{Variety of physical line interfaces:}

As stated before, ATM can be considered independent of the physical carrier it's placed upon. E3/DS3, E1/DS1, TAXI, STM-1, STS-3c, STM-16, ... the list of supported carriers grows almost every day. Again great for flexibility, but it adds additional complexity to the testing process. 


\section{High performance traffic capabilities:}

The roots of broadband are to be found in the quest for flexible admission of virtually unlimited bandwidth. Performing tests to verify if the network, or its separate nodes, deliver the switching capacity and throughput as expected, is imperative. Not only performance is the key issue here, but the reaction of the network to conditions of overload and congestion needs to be verifiable as well.

\section{Reliability:}

Broadband networks are not only famous for their performance, but also for their reliability and stability. Even this claim has to prove itself under stringent test conditions. Tests on behalf of long duration stability and error-free data transmission over the U-plane connections should not be absent in any self-respecting test-suite. Signaling alone (an aspect most covered in tests) does not make up an entire network, since the single most important purpose is to transport data.

\subsection{Suitable test tools}

Where it all boils down to is the availability of suitable test tools. Each of the previous aspects could be covered by a dedicated test tool. Regarding the earlier mentioned and inseparable "complexity-flexibility" twins, this would lead to a rather large pool of different test tools, each catering for a small, specialized domain of ATM testing. Make no mistake, these tools are necessary for those experts who are continuously working in these domains, in the form of research or development.

However, there is also a need for more universal test tools. These tools have to cover a larger area of testing, albeit not with the same in-depth attention to all possible domains as their dedicated counterparts provide. These tools would allow operators and manufacturers alike, to perform a significant part of their tests by means of one, easily usable device.

Ideally, such a device would adhere to the following requirements:

- Multi-interface support, to cover the wide variety of physical interfaces.

- User-alterable multi-protocol support, to counter problems with the inevitable protocol variants.

- Multi-role capabilities (conformance TTCN testing, inter-operability with narrowband, performance/BHCA/throughput testing).

- Provide for combined C/U-plane testing, not only to test signaling but error-free data transport as well.

- Non-intrusive line monitoring abilities.

- Suitable for stand-alone, LAN and long-distance testing.

- Modular expandability of all above mentioned requirements.

This versatility precludes practically all potential deficiencies as encountered in diverse problematics of broadband network testing. Pursuing this "broadband test utopia" is a goal that may never be achieved. However, the research results indicate that very well performing solutions can be found. 


\section{SEGMENTATION OF THE TARGET}

Clearly, due to the complexity of broadband network configurations and to the variety of ATM equipment, actual test tools have to provide the necessary versatility, power and flexibility to ensure that proper inter-operability between new ATM network components can be tested in a uniform and user-friendly way.

Research activities at Siemens Atea resulted in the development of the ATS ("ATM Traffic Simulator'), which is intended to fill in these requirements as much as possible.

\section{ATM TRAFFIC SIMULATOR}

\subsection{History}

End '93, when the research activities started, no appropriate ATM test equipment was found on the market. Therefore the research was started as described above. Of course when the needs are defined, an answer to these needs could prove the correctness. And if this answer turns out to be a good solution, it could also turn into a commercialisable product.

At first a solution was looked for in the adaptation of existing narrowband test equipment. However, to avoid being limited by the non-ATM based technology of the existing product, the effort was undertaken to start a new product from scratch, which is called ATS.

Commercial hardware for the rack and the processor module is used as a basis. The PC is taken as software development platform. A commercially available operating system is used on the test unit, complemented with an own operating system shell to implement the functions of a real time operating system.

In order to meet the demand of conceptual flexibility for allowing the operation with a user specified test scenario for the signaling layer under test, a Protocol Description Language (similar to textual SDL) is designed. The test scenario's can be modified in run time, without having to re-compile and re-link the complete load. From this test scenario, other layers/functions (f.i. data path testing) can be activated. To meet the demands for a test environment for conformance and interoperability tests, a complete TTCN workbench will be developed in the near future.

\subsection{System description}

\section{Introduction}

The ATS consists of a GUI (Graphical User Interface) and one or several SU's (System Units). The GUI software packet, running on a commercial PC, presents to the ATS user the interface to control the application software of the SU's. It is connected to the SU's via RS232 or Ethernet LAN interface, based on TCP/IP. The SU consists of an industrial PC cabinet with a CPU module, harddisk, network interface and currently up to 8 DIU's (Digital Interface Units). Each DIU contains the necessary hardware for testing one or more broadband interfaces. 


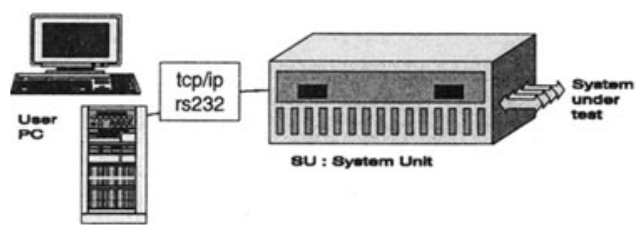

Figure 1 Local system configuration

\section{Hardware of the $S U$}

In today's maximum configuration, an SU consists of up to 8 interface boards (DIU's) to connect the system(s) under test, and one processing unit for the necessary test activities to be executed. The SU is VME compatible, the CPU board is PC compatible and uses an Intel processor. The system is transportable, the interfaces simple to exchange.

The DIU's interface to the system(s) under test, they are completely realized in hardware. The DIU blocks are available in different types depending on the interface requirements. Each DIU block contains a number of ATS test ports depending upon the DIU type (STM1 electrical, STM1 optical, E1/DS1, E3/DS3, ...). Each DIU consists of 2 cards : a BLM (Broadband Load Module) and a BIM (Broadband Interface Module). In B-ISDN, negotiation of bandwidth for the datapath may lead to different bandwidths per call. Therefore, each BLM contains programmable datapath testers, which are able to receive/send the required bandwidth. The BIM encapsulates/decapsulates the ATM cells. The ATM cells are exchanged between BLM/BIM via the standardized UTOPIA interface.

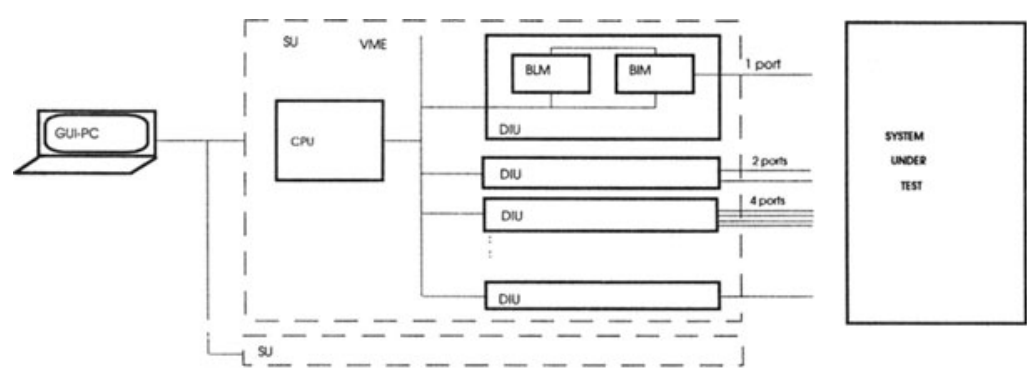

Figure 2 System architecture

Software

The software of the test equipment is mainly structured into the following blocks : on one hand the GUI and off-line tools for preparation of the test scenario's, on the other hand the test execution software on the SU.

\section{- GUI and Test Scenario Preparation SW:}


The GUI provides the access to the test system. It is responsible for the configuration, the administration and the supervision of the test units. It provides the capabilities to log events and diagnostic information on screen, printer or disk. Windows with pull-down menus, pop-up menus, window panes, toolbar, icon buttons and context sensitive help functions will guide the user. Off-line tools (i.e. the Sdtool) are offered for test scenario preparation.

\section{- Test execution SW:}

This software is responsible for the execution of the signaling testing, line monitoring, load testing and data connection testing.

The software part which is responsible for signaling, can be subdivided into 2 parts:

- A fixed part, representing the underlying protocol stack, is only defined for B-ISDN testing. It is based on AAL5.

- A programmable part, representing the signaling layer under test.

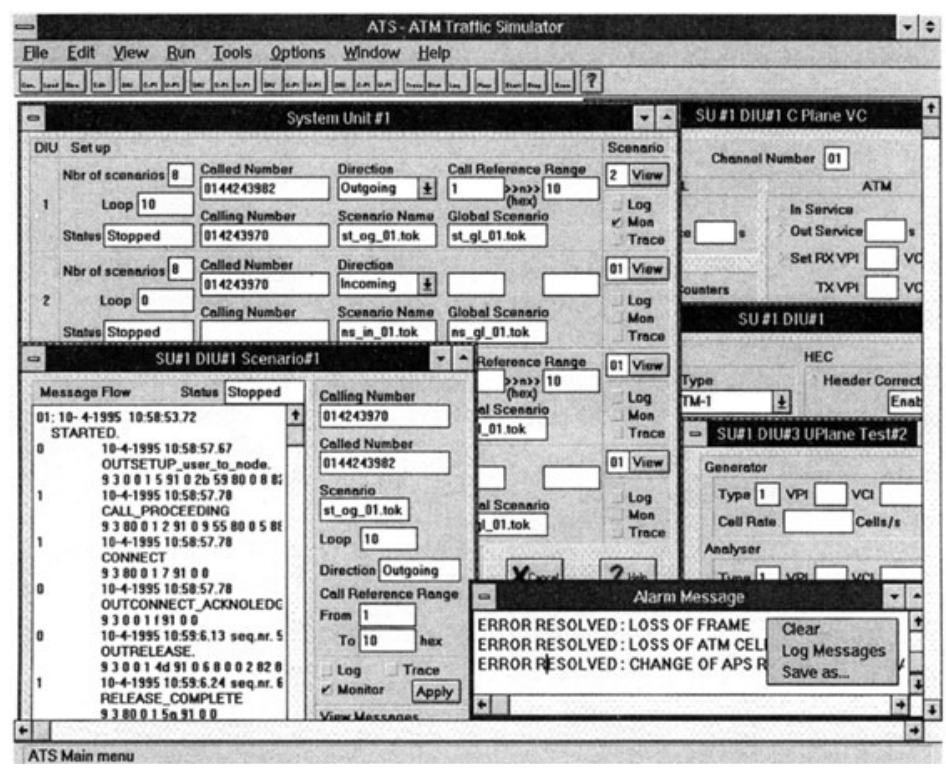

Figure 3 SU edit window on the GUI

The chosen operating system shell provides scheduling, timers, inter-process and inter-processor communication using the ACM (Asynchronous Communication Machine), MAE (Manageable Entity) and Timers concepts. It ensures the portability of the software on a different platform. The OMT (Object Modeling Technique) is applied as software engineering methodology, with $\mathrm{C}++$ as programming language. 


\subsection{Test configurations}

\section{Protocol and Load Test}

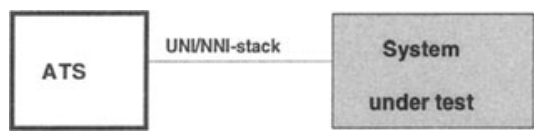

Figure 4 Protocol and Load testing configuration

The ATS is simulating a node and/or a terminal. The signaling protocol under test is simulated by replacing the implementation of a defined protocol layer by a test scenario. This scenario allows to test the protocol implementation of the SUT by enabling the tester to send out sequences of user defined protocol messages in order to verify the protocol behaviour in both, normal and error situations. In this way, the ATS can be used to perform manually conformance and interoperability tests.

\section{Line Monitoring}

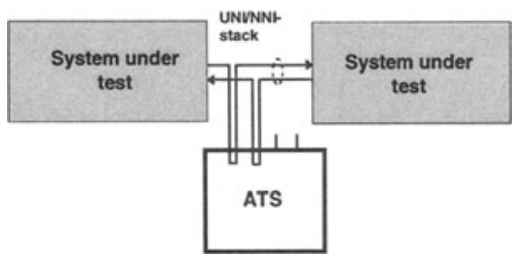

Figure 5 Line monitoring testing configuration

In line monitoring configuration, the ATS is looking at the signaling data passing on the line, without changing the data. The monitored data can be analysed on the fly or stored on the hard disk for a later off-line analysis.

\section{Data Path Testing}

The same test configuration that is used for protocol and load test applies. The testing of the data connections implies sending cells over the connections and checking the received information. Datapath testing can be triggered from within the scenarios (call related). It can also be used for testing PVC's (without any signaling relation).

\subsection{Description of the scenario concept}

Using a test scenario involves two separate phases, as shown in the next figure.

The first phase is the creation of a scenario which is done in the GUI. All means to create or change test programs with a behaviour fully specified by the user are provided. Elements of a 
protocol (incoming messages, outgoing messages, timers and state/event processing ) may be specified with a large degree of freedom.

The second phase is the set-up of the on-line form of one or more instantiations of a scenario created during the first phase.

\section{Phase-1: Creation of a test scenario.}

Test case generation starts with off-line test scenario preparation. Composing a scenario is making a behavior description in terms of scenario building components (SBC) i.e. messages, states, timers and actions, including the possibility of following certain branches in scenario dependent on conditions.

This phase is completely handled off-line. A test scenario is created/changed in three steps:

\section{- Step one}

A scenario script file ( filename.scn ), which is a text file, is created or changed using a set of rules described in the Protocol Description Language. The user is offered the option to define/change the name and/or contents of the SBC's.

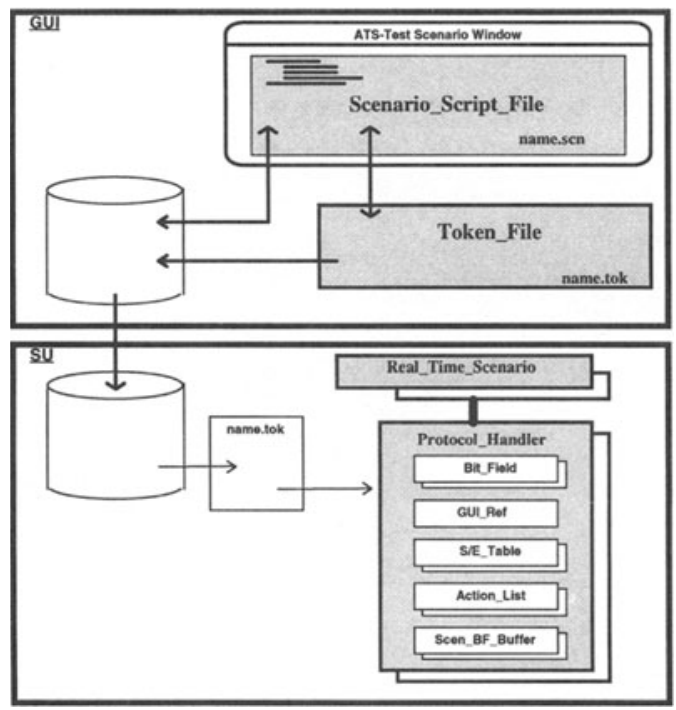

Figure 6 Scenario preparation

In the declaration of outgoing messages, there are no limits on the introduction of errors in the layout or content of messages. The actual content of a message sent in a running scenario can be dependent upon the contents of a received message. In the scenarios one can define several types of error injection. The ATS has a number of predefined SBC's giving the possibility of composing scenarios rather quickly. The SBC pool is structured according to the different 
protocols under test supported by the ATS (ATM Forum 3.1/4.0 (UNI), ITU Q.2931 (UNI), Q.2761-64 (NNI)).

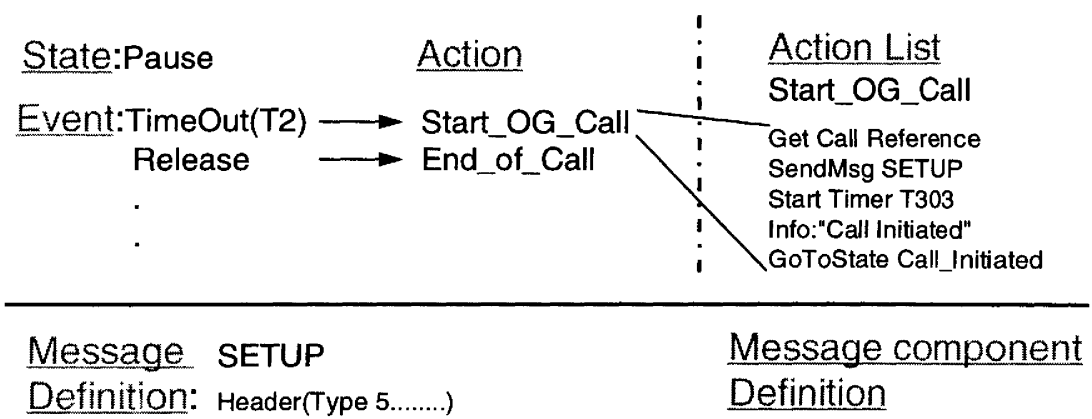
ATM_TRAFFIC_DESCRIPTOR
BB_BEARER_CAPABILITY
CALLED_PARTY
Every bitfield of the message
components is defined here.

Figure 7 The scenario definition can be subdivided into message definition, description of action lists, and a state/event description.

\section{- Step two}

A token file ( filename.tok ) is created with the Sdtool, ready for processing by the real-time software. During the conversion, several tests are performed to detect possible errors in the protocol specification. The conversion, done off-line, speeds up the processing on the real-time processor. The tools used for the processing of the textual representation are YACC and LEX. The first stage verifies the syntactical and semantic correctness of the input specification. During the second stage the textual description is disassembled into a set of inter-dependent data structures (bitfields, components, messages, states, timers and GUI conversion table). In the last stage the inter-dependent data structures are translated into a stream of tokens representing the necessary real time objects to simulate the specified protocol. In here you can find all necessary information how to identify a message, which bitfields must be checked and which actions must be taken. The first and second step are processed simultaneously, while the token generation starts at the end of file of the textual description.

A scenario token file has several parts in a specific order. The order of appearance is essential for the setup of the real time scenario.

\section{- Step three}

The created/changed token file ( filename.tok ) is copied from the DIU disk to the SU disk. 


\section{Phase-2: Set-up of an on-line scenario.}

The SU receives the instructions for the set-up of one or more on-line scenario's in one or more DIU's from the GUI. An on-line scenario is the executable form of a scenario token file. A simplified form of on-line scenario's is presented by the figure below. As one can see there are two parts involved in on-line scenario's : the RTScenario (Real Time Scenario) and a ProtocolHandler. A RTScenario is created for each simultaneous instantiation of a scenario and contains all information which is specific for each running copy of a scenario.

A ProtocolHandler is created with all the information of a token file and contains three types of building elements : buffers, action trees and state/events:

- All data of a running scenario, is located in buffers.

- An action tree is installed and executed as a queue of different actions where each action in a tree returns a reference to the next action or an end indication.

- A state/event is created for each state of a scenario. Each state/event is programmed with the information to recognise the user specified incoming messages and events, as well as with the references to the action tree specified for each case.

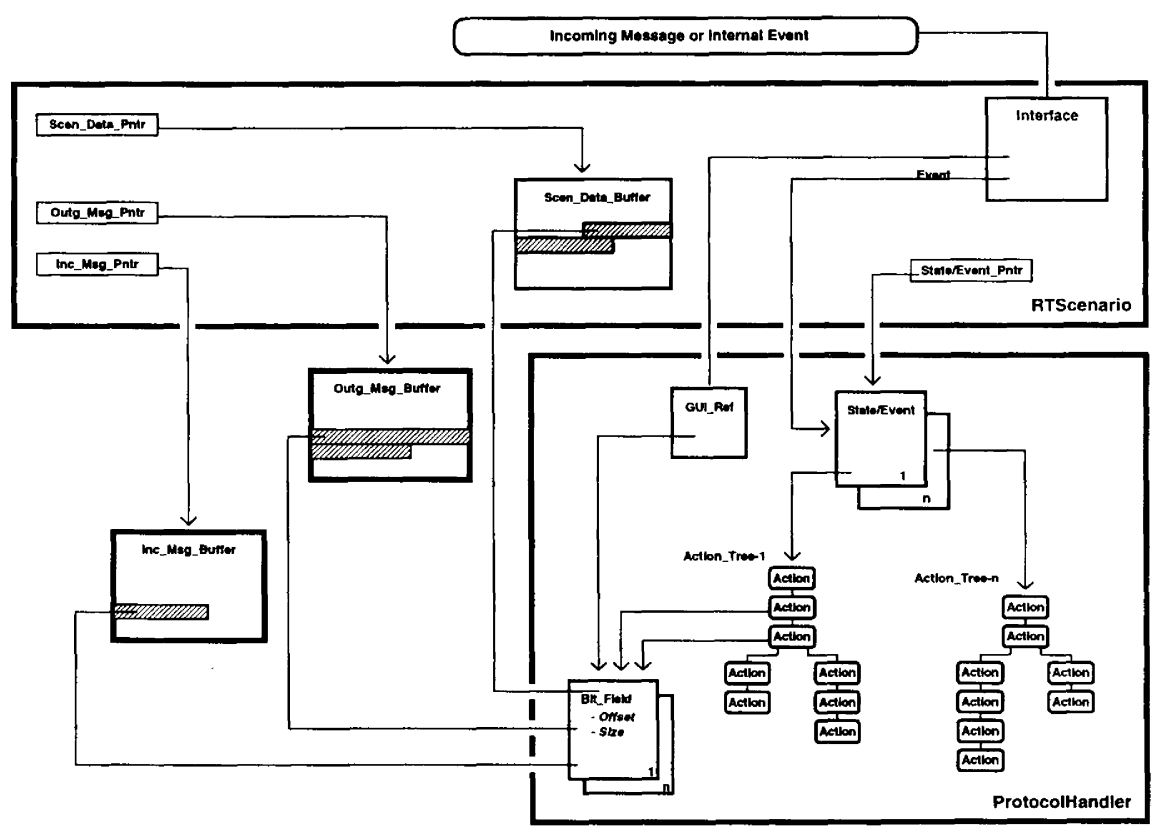

Figure 8 Setup of a real time scenario 


\section{CONCLUSION}

The lack of versatile test equipment on the ATM market, triggered research activities at Siemens Atea. The subsequent "R\&D"-effort spawned the first prototype of the ATS, which in its turn has matured and proven itself as a viable test tool for ATM networks. From the earliest stages on, the most important key points in the development of the ATS have been flexibility, expandability and user-friendliness. A test tool that is too rigid to cater for the evolving needs of the systems it is destined to verify, is not worth the resources it dissipated. Neither should the ease of use be dwarfed by the power the device actually harbours.

The ATS does not aspire to be everything to everyone in the world of ATM testing, but it does offer a workable solution for significant areas of ATM network testing.

\section{ABBREVIATIONS}

$\begin{array}{ll}\text { ATM } & \text { Asynchronous Transfer Mode } \\ \text { ATS } & \text { ATM Traffic Simulator } \\ \text { DIU } & \text { Digital Interface Unit } \\ \text { DS1 } & \text { Digital Signal 1, method for digital transmission at } 1.5 \mathrm{Mbit} / \mathrm{s} \\ \text { DS3 } & \text { Digital Signal 3, method for digital transmission at } 44 \mathrm{Mbit} / \mathrm{s} \\ \text { E1 } & \text { ITU standard for digital transmission at 2 Mbit/s } \\ \text { E3 } & \text { ITU standard for digital transmission at 34 Mbit/s } \\ \text { GUI } & \text { Graphical User Interface } \\ \text { LAN } & \text { Local Area Network } \\ \text { NNI } & \text { Network Node Interface } \\ \text { PDL } & \text { Protocol Description Language } \\ \text { PVC } & \text { Permanent Virtual Channel } \\ \text { SBC } & \text { Scenario Building Component } \\ \text { SDL } & \text { Specification and Description Language } \\ \text { STM-1 } & \text { Synchronous Transport Module level 1, a 155Mbit/s interface } \\ \text { SU } & \text { System Unit } \\ \text { SUT } & \text { System Under Test } \\ \text { UNI } & \text { User to Network Interface } \\ \text { UTOPIA } & \text { Universal Test and Operations Physical Interface for ATM }\end{array}$

\section{REFERENCES}

- Martin de Prycker (1991), Asynchronous Transfer Mode, solution for broadband ISDN, ISBN 0-13-053513-3

- Daniel Minoli, George Dobrowski (1995), Principles of signaling for cell relay and frame relay, ISBN 0-89006-708-2

The ATM Forum (1993), ATM User-Network Interface Specification version 3.0, Prentice Hall PTR, ISBN 0-13-225863-3 


\section{BIOGRAPHY}

- Ing. Luc Vandevenne Luc Vandevenne graduated as industrial engineer electronics in 1989 at the K.I.H.L. in Diepenbeek. His professional carreer started at Siemens Atea as protocol test engineer. From 1992 he coordinated the system test process for several projects (ISDN and GSM) and steered the introduction in the field of several releases in this area. Since 1995 he is project manager for broadband test equipment.

- Ir. Beatrix De Sutter

Bea De Sutter graduated as civil engineer physics at University of Ghent in 1989. In 1989 she did research in nuclear physics at KFA in Julich (Germany). In 1990 she joined Siemens Atea as protocol designer in the area of SCCP and TCAP. From 1995 on she moved to broadband protocol design and now coordinates the activities in the ACTS INSIGNIA project for Siemens Atea.

- Ing. Erik Goossens

Erik Goossens graduated as industrial engineer electronics (chip design) at K.I.H.L Diepenbeek in 1994. Immediately after his studies he joined Siemens Atea as sytem verification engineer. Now he is involved in the testing of the Belgian releases for narrowband telecommunication systems. 